\title{
On sign changes of tempered distributions having a spectral gap at the origin
}

\section{Sur les changements du signe de distributions tempérées ayant une lacune spectrale à l'origine}

\author{
Iossif Ostrovskii ${ }^{\mathrm{a}, \mathrm{b}}$, Alexander Ulanovskii ${ }^{\mathrm{c}}$ \\ a Department of Mathematics, Bilkent University, 06533 Bilkent, Ankara, Turkey \\ ${ }^{\mathrm{b}}$ Verkin Institute for Low Temperature Physics and Engineering, 61103 Kharkov, Ukraine \\ ${ }^{\mathrm{c}}$ Stavanger University College, PO Box 2557, Ullandhaug, 4091 Stavanger, Norway
}

Received 6 January 2003; accepted 14 January 2003

Presented by Jean-Pierre Kahane

\begin{abstract}
It is known that if a real finite Borel measure has a spectral gap at the origin then either it must have many sign changes or it is zero identically. Assume the Fourier transform of a real temperate distribution agrees in a neighborhood of the origin with the sum of an analytic function and a lacunary trigonometric series. We conjecture that either the distribution must have many sign changes or the Fourier transform agrees with the sum on the whole line. The Note contains some results related to the conjecture. In particular, our results imply that a real temperate measure having spectral gap at the origin must have many oscillations with large amplitudes. To cite this article: I. Ostrovskii, A. Ulanovskii, C. R. Acad. Sci. Paris, Ser. I 336 (2003). C 2003 Académie des sciences/Éditions scientifiques et médicales Elsevier SAS. All rights reserved.
\end{abstract}

\section{Résumé}

Il est connu que si une mesure de Borel réelle a une lacune spectrale à l'origine, alors ou la mesure doit avoir beaucoup de changements du signe ou elle est zéro identiquement. Supposons que la transformée de Fourier d'une distribution tempérée réelle coïncide dans un voisinage de l'origine avec la somme d'une fonction analytique et d'une série trigonometrique lacunaire. Nous conjecturons que ou elle coïncide avec la somme sur toute la ligne réelle ou la distribution doit avoir beaucoup de changements du signe. La Note contient quelques résultats reliés à la conjecture. En particulier les résultats impliquent qu'une distribution tempérée réelle ayant une lacune spectrale à l'origine doit avoir beaucoup d'oscillations d'une grande amplitude. Pour citer cet article : I. Ostrovskii, A. Ulanovskii, C. R. Acad. Sci. Paris, Ser. I 336 (2003).

(C) 2003 Académie des sciences/Éditions scientifiques et médicales Elsevier SAS. Tous droits réservés.

E-mail addresses: iossif@fen.bilkent.edu.tr, ostrovskii@ilt.kharkov.ua (I. Ostrovskii), Alexander.Ulanovskii@tn.his.no (A. Ulanovskii). 


\section{Version française abrégée}

Le fait suivant est bien connu en Analyse Harmonique : Si une mesure réelle sur $\mathbf{R}$ a une lacune spectrale à l'origine, son signe doit avoir beaucoup de changements. Le resultat suivant est dû à Krein et Levin ([3], Appendix 2, Theorem 5):

Théorème de Krein-Levin. Soit $\mu$ une mesure de Borel réelle et finie sur $\mathbf{R}$. Supposons que $\hat{\mu}=0$ sur $(-a, a)$. Alors on a l'inégalité (2), $n_{\mu}(-r, r)$ étant le nombre de changements du signe de $\mu$ sur $(-r, r)$ défini par (1).

Pour des autres résultats et références, voir [5,7], [8, Section 5] and [9].

Nous conjecturons que le même phénomène se présente dans des situations plus générales : supposons que la transformée de Fourier $\hat{f}$ d'une distribution tempérée réelle $f$ coïncide dans un voisinage de l'origine avec la somme d'une fonction analytique et d'une série trigonométrique lacunaire. Si la représentation n'est pas valable sur toute la ligne réelle, le signe de $f$ doit avoir beaucoup de changements. Ce travail contient quelques résultats qui confirment la conjecture.

Nous renvoyons au livre de Hörmander [1] pour la définition des distributions tempérées et de leurs propriétés fondamentales.

Une distribution tempérée réelle $f$ étant donnée, on peut définir une suite $Z_{f}^{+}$de points positifs (avec des multiplicités) où $f$ change de signe. Par définition, $x \in Z_{f}^{+}$si la fonction $n_{f}((0, r))$ definie par (4) est discontinue en $x$. On désigne par $D^{*}\left(Z_{f}^{+}\right)$la densité extérieure de Beurling et Malliavin (voir [2], Chapitre 9) de $Z_{f}^{+}$. Nous disons qu'une série trigonométrique $\sum_{n} c_{n} \mathrm{e}^{\mathrm{i} x_{n} t}$, où $\sum_{n}\left|c_{n}\right|<\infty$, est lacunaire si $D^{*}\left(\left\{x_{n}\right\}\right)=0$.

Théorème 0.1. Soit $f$ une distribution tempérée réelle. Supposons que la restriction de sa transformée de Fourier $\hat{f} \operatorname{sur}(-a, a)$ a une représentation $\hat{f}(t)=G(t)+H(t)$, où $G$ est la valeur frontière d'une fonction analytique dans une bande $\{0<\Im t<R\}$ et continue dans sa fermeture, et $H$ est une série lacunaire. Alors ou cette représentation est valable sur toute la ligne réelle ou $D^{*}\left(Z_{f}^{+}\right) \geqslant a / \pi$.

Comme conséquence immédiate on a :

Corollaire. Supposons que $f$ soit une distribution tempérée réelle qui vérifie $D^{*}\left(Z_{f}^{+}\right)<a / \pi$, et qu'une fonction $G$ soit analytique dans une bande $\{0<\Im t<R\}$ et continue dans sa fermeture. Si $\hat{f}(t)=G(t)$ sur $(-a, a)$, alors $\hat{f}=G$.

Le principe suivant est classique en Analyse Harmonique : Supposons que $\mu$ soit une mesure de Borel finie et positive. Si $\hat{\mu}$ est lisse à l'origine, alors elle est lisse sur toute la ligne réelle. Le corollaire prolonge cela de la manière suivante : si une distribution tempérée réelle a «peu» de changements de signe et sa transformée de Fourier est « lisse» (= la valeur frontière d'une fonction analytique) sur $(-a, a)$, alors elle est «lisse» sur toute la ligne réelle. Observons que l'exemple $f=\sin a x$ montre qu'on ne peut pas relâcher la restriction $D^{*}\left(Z_{f}^{+}\right)<a / \pi$ dans le corollaire.

Le Théorème 0.1 et corollaire utilisent la densité extérieure de Beurling et Malliavin qui est du type d'une densité supérieure. Cependent le théorème de Krein-Levin utilise la densité moyenne. Le résultat suivant généralise le théorème de Krein-Levin pour les mesures infinies en tenant compte pas seulement du $n_{\mu}(-r, r)$ mais aussi du $n_{\mu}(0, r)$ :

Théorème 0.2. Soit $\mu \neq 0$ une mesure de Borel réelle qui vérifie (5). Si $\hat{\mu}=0$ sur $(-a, a)$, alors les inégalités (6) et (7) sont valables. 


\section{Introduction and results}

Let $\mu$ be a finite Borel measure on the real line, and denote by $\hat{\mu}$ its Fourier transform

$$
\hat{\mu}(t)=\int_{-\infty}^{\infty} \mathrm{e}^{-\mathrm{i} t x} \mathrm{~d} \mu(x) .
$$

We say that $\mu$ has a spectral gap on $(-a, a)$ if $\hat{\mu}(t)=0$ for $-a<t<a$. We define the number of sign changes of $\mu$ on an interval $I$ as follows:

$$
n_{\mu}(I)=\min \{\operatorname{deg} P: P \text { is a polynomial such that } P \mu \geqslant 0 \text { on } I\} \text {. }
$$

We set $n_{\mu}(I)=\infty$, if there is no polynomial $P$ such that $P \mu \geqslant 0$ on $I$.

The following fact is known in Harmonic Analysis: if a real measure on $\mathbf{R}$ has a spectral gap at the origin then it must have many sign changes. The following result is due to Krein and Levin ([3], Appendix 2, Theorem 5):

Krein-Levin Theorem. Assume that a real finite Borel measure $\mu$ has a spectral gap on $(-a, a)$. Then

$$
\liminf _{R \rightarrow \infty}\left(\int_{1}^{R} \frac{n_{\mu}(-r, r)}{r} \mathrm{~d} r-\frac{2 a}{\pi} R\right)>-\infty
$$

It was shown by Logan in 1965 that if a nontrivial real $L_{\infty}$-function $f$ is nonnegative on $[0, \infty)$, then it cannot have a spectral gap on any interval $(-a, a)$. Another result of Logan [5] states that if $\hat{f}(x)=0$ on $(-a, a)$ and the support of $\hat{f}$ is compact (which means that $f$ is an entire function of exponential type) then the number $n_{f}(0, r)$ of sign changes on $(0, r)$ of $f$ satisfies $\liminf _{r \rightarrow \infty} n_{f}(0, r) / r \geqslant a / \pi$. Recently Eremenko and Novikov informed us that they have proved this estimate in much more general situation. Some other results in this direction and open problems can be found in [7], [8, Section 5] and [9].

We conjecture that the same phenomenon occurs in more general situations: assume that the Fourier transform $\hat{f}$ of a real temperate distribution $f$ agrees in a neighborhood of the origin with the sum of an analytic function and a lacunary series. If this representation does not hold on the whole real line, then $f$ must have many sign changes. We shall prove several results confirming this conjecture. A corollary is that the real distributions having spectral gap at the origin must have many oscillations with large amplitudes.

We refer the reader to Hörmander's book [1] for the definition of temperate distributions (t.d.) and their basic properties.

We shall use the Beurling-Malliavin exterior density $D^{*}$ to estimate the size of a sequence. Let $Z$ be a sequence of real numbers where we allow repetitions. There are several equivalent definitions of $D^{*}$ (see [2], Chapter 9). If $Z \subset(0, \infty)$, one can define $D^{*}(Z)$ as the infimum of the positive numbers $A$ for which there exist positive sequences $\Omega \supseteq Z$ with

$$
\int_{0}^{\infty} \frac{\left|n_{\Omega}(0, t)-A t\right|}{1+t^{2}} \mathrm{~d} t<\infty
$$

Here $n_{\Omega}(0, t)$ is the number of points of $\Omega$ on $(0, t)$ (see [2], p. 85). Observe that $Z$ is a subsequence of $\Omega$, that is every element of $Z$ occurs in $\Omega$ at least as many times as it does in $Z$. In general, $D^{*}(Z)=$ $\max \left\{D^{*}\left(Z^{+}\right), D^{*}\left(Z^{-}\right)\right\}$, where $Z^{+}=Z \cap[0, \infty)$ and $Z^{-}=Z \cap(-\infty, 0)$. Let $B_{a}$ denote the class of all nontrivial entire functions $\varphi$ of exponential type $<a$ which are bounded on the real line. According to Beurling and Malliavin, the density $D^{*}(Z)$ is equal to the infimum of $a / \pi$, where $a>0$ is such that there exists $\varphi \in B_{a}$ which vanishes at every point $x \in Z$ with given multiplicity. If such functions $\varphi$ do not exist, then $D^{*}(Z)=\infty$. In particular, 
$D^{*}(Z)=0$ if and only if for any $a>0$ there is a function $\varphi \in B_{a}$ which vanishes at every point $x \in Z$ with given multiplicity.

We shall deal with t.d. $f$ whose Fourier transform $\hat{f}$ admits analytic continuation in the following sense:

Definition. We say that a t.d. $\hat{f}$ is half-analytic on $(-a, a)$ if $\hat{f}$ is the boundary value in $\mathcal{S}^{\prime}$-topology of a function which is analytic in some strip $\{t: 0<\Im t<R\}$, continuous in $\{t: 0<\Im t<R\} \cup(-a, a)$ and is $\mathrm{O}\left(|t|^{N}\right)$ with some $N$ as $t \rightarrow \infty$ in any strip $\left\{t: 0<R_{1} \leqslant \Im t \leqslant R_{2}<R\right\}, 0<R_{1}<R_{2}<R$.

Observe that if $\hat{f}$ is half-analytic on $(-a, a)$ and $\hat{f}=0$ in some neighborhood of the origin then $f=0$ identically. Observe also that if $\hat{f}$ is the boundary value of a function analytic in a strip $\{t: 0<\Im t<R\}$ and continuous in its closure then $\hat{f}$ is half-analytic on the whole line.

It was established in [6] (see Theorem A below) that if a t.d. $f$ is nonnegative on some half-line $(b, \infty)$ then it cannot have a spectral gap at the origin unless $f=0$. The following observation is trivial: if $\hat{f}=0$ on $(-a, a)$, then for any $\varphi \in B_{a}$ the product $\varphi \cdot f$ has a spectral gap at the origin. Hence, the product $\varphi \cdot f$ cannot be nonnegative on a half-line unless $f=0$. We generalize this as follows:

Theorem 1.1. Let $f$ be a nontrivial t.d. having spectral gap on $(-a, a), g$ be a t.d. such that $\hat{g}$ half-analytic on $(-a, a)$, and let $h$ be a t.d. of finite order whose support is discrete and satisfies $D^{*}(\operatorname{supp} h)=0$. Then there is no function $\varphi \in B_{a}$ such that the distribution $\varphi \cdot(f+g+h)$ is nonnegative on some half-line $(b, \infty)$.

Suppose $f$ is a real t.d., and $I$ is an interval. One may define the number of sign changes of $f$ on $I$ as in (1):

$$
n_{f}(I)=\min \{\operatorname{deg} P: P \text { is a polynomial such that } P \cdot f \geqslant 0 \text { on } I\} \text {. }
$$

We set $n_{f}(I)=\infty$ if such polynomials do not exist. Let us fix a number $b$ and let $r>b$. The function $n_{f}(b, r)$ is integer-valued. One can now define the sequence $Z_{f}(b, \infty)$ of the sign changes of $f$ on $(b, \infty)$ as the sequence of all points $x>b$ where $n_{f}(b, r)$ has jumps, the multiplicity of $x$ being the size of the jump.

Theorem 1.1 and some properties of the density $D^{*}$ imply

Theorem 1.2. Let distributions $f, g$ and $h$ satisfy the assumptions of Theorem 1.1. Then $D^{*}\left(Z_{f+g+h}(b, \infty)\right) \geqslant a / \pi$ for any $b$.

Observe that if the t.d. $g$ is in certain sense 'small' as $x \rightarrow \infty$, then $\hat{g}$ can be analytically continued to some strip in the upper half-plane. In this case, one can view the sum $f+g+h$ as a 'perturbation' of the t.d. $f$. Here $g$ is 'small' while $h$ is 'sparse'. Hence, if $f$ has spectral gap on $(-a, a)$, any such perturbation must have many sign changes on any fixed half-line $(b, \infty)$. This can be viewed as an information about the amplitude of the oscillations of $f$.

Let us say that a trigonometric series $\sum_{n} c_{n} \mathrm{e}^{\mathrm{i} x_{n} t}$, where $\sum_{n}\left|c_{n}\right|<\infty$, is lacunary if $D^{*}\left(\left\{x_{n}\right\}\right)=0$. If the distribution $h$ in Theorem 1.2 is a finite measure then $\hat{h}$ is a lacunary series. Theorem 1.2 implies the following

Corollary 1. Suppose $f$ is a nontrivial real t.d. such that the restriction of its Fourier transform $\hat{f}$ to $(-a, a)$ admits a representation: $\hat{f}(t)=G(t)+H(t)$, where $G$ is the boundary value of a function analytic in a strip $\{0<\Im t<R\}$ and continuous in its closure, and $H$ is a lacunary trigonometric series. Then either this representation holds on the whole real line or $D^{*}\left(Z_{f}(b, \infty)\right) \geqslant a / \pi$, for any $b$.

The following principle is classical in Harmonic Analysis: suppose $\mu$ is a positive finite Borel measure. If $\hat{\mu}$ is 'smooth' at the origin then it is 'smooth' on the whole real line (see, e.g., [4], Chapter 2). Corollary 1 implies

Corollary 2. Let a $>0, f$ be a nontrivial real t.d. satisfying $D^{*}\left(Z_{f}(b, \infty)\right)<a / \pi$, and let $G$ be a function analytic in a strip $\{0<\Im t<R\}$ and continuous in its closure. If $\hat{f}=G$ on $(-a, a)$ then $\hat{f}=G$. 
This result extends the principle above by showing that if a real t.d. $f$ has a 'small number' of sign changes on $(b, \infty)$, and its Fourier transform is 'smooth' (= boundary values of analytic function) on $(-a, a)$ then it is 'smooth' on the whole real line. Observe that the example $f_{1}=\sin a x$ shows that the restriction $D^{*}\left(Z_{f}(b, \infty)\right)<a / \pi$ in Corollary 2 cannot be relaxed.

The above Theorem 1.2 deals with the Beurling and Malliavin exterior density which is a kind of upper density. Meanwhile the Krein-Levin theorem deals with the averaged density. The following result generalizes KreinLevin theorem to infinite measures and takes into account not only $n_{\mu}(-r, r)$ but also $n_{\mu}(0, r)$ :

Theorem 1.3. Let $\mu \neq 0$ be a real Borel measure satisfying

$$
\int_{-\infty}^{\infty} \frac{\mathrm{d}|\mu|(t)}{1+t^{2}}<\infty .
$$

If $\hat{\mu}=0$ on $(-a, a)$ then the inequalities hold:

$$
\begin{aligned}
& \liminf _{R \rightarrow \infty}\left\{\int_{1}^{R}\left(\frac{1}{t^{2}}+\frac{1}{R^{2}}\right) n_{\mu}(0, t) \mathrm{d} t-\frac{a}{\pi} \log R\right\}>0 ; \\
& \liminf _{R \rightarrow \infty}\left\{\int_{1}^{R} \frac{n_{\mu}(-t, t)}{t} \mathrm{~d} t-\frac{2 a}{\pi} R+3 \log R\right\}>0 .
\end{aligned}
$$

\section{Proofs of Theorems 1.1 and 1.2}

We shall need the following result:

Theorem A ([6], Theorem 3). Let $f$ be a t.d. nonnegative on some half-line $(b, \infty)$. Assume that its Fourier transform $\hat{f}$ agrees on $(-\delta, \delta)$ with a function analytic in a rectangle $\{t: 0<\Im t<R,|\Re t|<\delta\}$ and continuous in its closure. Then $\hat{f}$ is half-analytic on $(-\delta, \delta)$.

Proof of Theorem 1.1. Assume that a real t.d. $f$ has a spectral gap on $(-a, a)$, and that t.d. $g$ and $h$ satisfy the assumptions of Theorem 1.1. Assume further that $\varphi(f+g+h) \geqslant 0$ on $(b, \infty)$, for some nontrivial function $\varphi \in B_{a}$. To prove Theorem 1.1 we have to show that $f=0$.

By the definition of class $B_{a}$ there is a positive number $\varepsilon$ such that the type of $\varphi$ is less than $a-3 \varepsilon$. Let an integer $N$ be larger than the order of distribution $h$. Since $D^{*}(\operatorname{supp} h)=0$, there exists a real function $\psi \in B_{\varepsilon}$ which vanishes at every $x \in \operatorname{supp} h$ with the multiplicity $\geqslant N$. It follows that $\psi h=0$. Set $\varphi_{1}=\varphi \psi^{2}$. Clearly, the distribution $\varphi_{1} \cdot(f+g+h)=\psi^{2} \varphi \cdot(f+g)$ is nonnegative on $(b, \infty)$. The type of $\varphi_{1}$ is less than $a-\varepsilon$, so that the support of its Fourier transform $\hat{\varphi}_{1}$ belongs to $(-a+\varepsilon, a-\varepsilon)$. It follows that $\hat{\varphi}_{1} * \hat{f}=0$ on $(-\varepsilon, \varepsilon)$. Also, since $\hat{g}$ is half-analytic on $(-a, a)$, the convolution $\hat{\varphi}_{1} * \hat{g}$ is half-analytic on $(-\varepsilon, \varepsilon)$. We see that the distribution $\varphi_{1} \cdot(f+g)$ satisfies the assumptions of Theorem $\mathrm{A}$, and so the product $\hat{\varphi}_{1}(\hat{f}+\hat{g})$ is half-analytic on $(-\varepsilon, \varepsilon)$. Hence, the same is true for $\varphi_{1} \cdot f$. Since the convolution $\hat{\varphi}_{1} * \hat{f}$ vanishes on $(-\varepsilon, \varepsilon)$, we get $\hat{\varphi}_{1} * \hat{f}=0$. Write $\hat{f}=F_{-}+F_{+}$where $F_{-}$ and $F_{+}$are the restrictions of $\hat{f}$ to the negative and positive half-lines, respectively. We have supp $F_{-} \subseteq(-\infty,-a]$ and $\operatorname{supp} F_{+} \subseteq[a,-\infty)$. Since $\operatorname{supp} \hat{\varphi}_{1} \subset(-a+\varepsilon, a-\varepsilon)$, we see that $\operatorname{supp} \hat{\varphi}_{1} * F_{-} \cap \operatorname{supp} \hat{\varphi}_{1} * F_{+}=\emptyset$. This gives $\hat{\varphi}_{1} * F_{-}=0$ and $\hat{\varphi}_{1} * F_{+}=0$. Hence, $\varphi_{1} \widehat{F}_{-}=\varphi_{1} \widehat{F}_{+}=0$. However, $\varphi_{1}$ is an entire function, and both $\widehat{F}_{-}$ and $\widehat{F}_{+}$are boundary values in $\mathcal{S}^{\prime}$-topology of functions analytic in the upper (lower) half-planes, respectively. We conclude that $\widehat{F}_{-}=\widehat{F}_{+}=0$, so that $f=0$. 
Proof of Theorem 1.2. Given a sequence of $S$ with repetitions, we denote by $m_{S}(x)$ the multiplicity (number of occurrences) of the number $x$ in $S$. We set $m_{S}(x)=0$ if $x \notin S$. Let us also denote by $n_{S}(0, r)$ the number of points (counted with multiplicities) of $S$ in $(0, r)$, and by $\mathbf{N}_{0}$ the set of nonnegative integers.

Suppose that t.d. $f, g$ and $h$ satisfy the assumptions of Theorem 1.1. Set $Z=Z_{f+g+h}(b, \infty)$. To prove Theorem 1.2 we have to show that $D^{*}(Z) \geqslant a / \pi$ for every $b$. Assume that this is not true. Then there is a number $\varepsilon>0$ such that $D^{*}(Z)<a / \pi-2 \varepsilon$ for some $b>0$. It follows that there exists $\psi \in B_{a-2 \varepsilon}$ which vanishes at each $x \in Z$ and $m_{S}(x)-m_{Z}(x) \geqslant 0$ for all $x>0$, where $S$ is the sequence of positive zeros of $\psi$. Let $\left\{x_{1}<x_{2}<; \ldots\right\}$ be the set of all $x_{k} \in S$ such that $m_{S}\left(x_{k}\right)-m_{Z}\left(x_{k}\right)-1 \in 2 \mathbf{N}_{\mathbf{0}}$. Set

$$
P(z)=\prod_{k} \frac{1-z / x_{k}}{1-z / x_{k+1}} .
$$

Simple calculations show that $|P(x \pm \mathrm{i})|=\mathrm{O}\left(|x|^{2}\right)$ as $|x| \rightarrow \infty$. Set

$$
\varphi(z)=\psi(z) P(z) \frac{\sin ^{2} \varepsilon z}{z^{2}} .
$$

Since $\psi$ vanishes at the poles of $P$, standard reasonings show that $\varphi$ is entire function of finite exponential type. Clearly, the type of $\varphi$ is less than $a$. Moreover, since $\psi(x \pm \mathrm{i})$ is bounded, the same is true for $\varphi$. Applying to $\varphi$ the

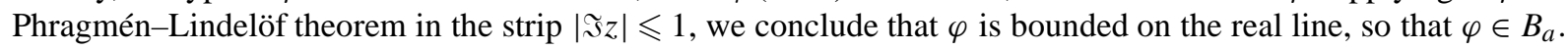
The sequence $S^{*}$ of the positive zeros of $\varphi$ satisfies $m_{S^{*}}\left(x_{j}\right)-m_{Z}\left(x_{j}\right) \in 2 \mathbf{N}_{\mathbf{0}}$ for all $x>0$. By the definition of $Z$, this gives $\varphi \cdot(f+g+h) \geqslant 0$ on $(b, \infty)$. This contradicts to Theorem 1.1, which proves Theorem 1.2.

\section{Acknowledgement}

The authors thank Prof. M. Sodin for his encouragement and valuable comments. The research was done during a visit of the second named author to Bilkent University at Ankara. This visit was supported by the Scientific and Technical Research Council of Turkey (TÜBITAK).

\section{References}

[1] L. Hörmander, The Analysis of Linear Partial Differential Operators, I, Springer-Verlag, Berlin, 1983.

[2] P. Koosis, The Logarithmic Integral, Vol. 2, Cambridge University Press, 1992.

[3] B.Ja. Levin, Distribution of Zeros of Entire Functions, American Mathematical Society, Providence, RI, 1980.

[4] Ju.V. Linnik, I.V. Ostrovskii, Decomposition of Random Variables and Vectors, American Mathematical Society, Providence, RI, 1977.

[5] B.F. Logan, Jr., Properties of high-pass signals, Thesis, Electrical Engineering Department, Columbia University, New York, 1965.

[6] I.V. Ostrovskii, A. Ulanovskii, On the Lévy-Raikov-Marcinkiewicz theorem, C. R. Acad. Sci. Paris, submitted.

[7] H.S. Shapiro, Functions with spectral gap, Bull. Amer. Math. Soc. 79 (2) (1973) 354-360.

[8] H.S. Shapiro, Notes on a theorem of Baouendi and Rothschild, Exposition. Math. 13 (1995) 247-275.

[9] H.S. Shapiro, Rate of decay of convolution vs. frequency and sign changes, in: J.S. Byrnes (Ed.), Twentieth Century Harmonic Analysis a Celebration, Kluwer Academic, Dordrecht, 2001, pp. 383-384. 